\title{
Splenic epidermoid cysts presenting as an acute abdomen
}

\author{
Mark Thomas and Bola Taiwo
}

Hemel Hempstead General Hospital, Hillfield Road, Hemel Hempstead, Hertfordshire HP2 4AD, UK

\author{
Summary: Two patients with splenic epidermoid cysts who presented as acute abdominal emergencies \\ are reported.
}

\section{Introduction}

Splenic cysts are rare, especially in western countries. Worldwide, parasitic splenic cysts are more frequently encountered with Echinococcus granulosa being the most commonly implicated species. ${ }^{1}$ The mode of presentation varies according to age group. In children, intermittent colicky abdominal pain in the left hypochondrium with a tender palpable mass is usually the way splenic cysts present. ${ }^{2}$ In adults it is usually picked up incidentally on abdominal ultrasonography. In some regions of the world where $E$. granulosa is epidemic, however, presentation may be through complications such as infection or rupture. ${ }^{3}$

The concurrent presentation of two cases of proven splenic epidermoid cysts as an acute abdomen at short intervals to a district general hospital is unusual. We therefore hope to highlight this condition in the differential diagnosis of the surgical abdominal emergency.

\section{Case reports}

Case 1

A 20 year old woman presented with a one week history of sudden onset of left-sided abdominal pain. The pain was constant in nature with a dragging feeling made worse by eating and breathing deeply. Her appetite had been poor and she had vomited once.

On examination she was obviously in pain. There was generalized abdominal tenderness especially in the left hypochondrium where the spleen was palpable. She underwent laparotomy and splenectomy. Several spleneculi were found and left behind. The spleen contained a $13 \mathrm{~cm}$ diameter cyst and weighed $1,169 \mathrm{~g}$. The histology was that of epidermoid cyst (Figure 1).

Correspondence: B. Taiwo, F.R.C.S., John Coupland Hospital, Ropery Road, Gainsborough, Lincs DN21 2TJ Accepted: 23 September 1993
Case 2

A 25 year old woman, 4 months post-delivery, presented with the sudden onset of left abdominal pain. She had noticed a painless swelling in the abdomen just after her confinement which had been getting progressively bigger. She had experienced only slight discomfort over the swelling before her acute presentation to hospital.

On examination she had a tender splenic mass in the left upper quadrant $12 \mathrm{~cm}$ below the costal margin.

Full blood count and bone marrow aspiration were normal. Abdominal ultrasound showed a large cyst in the spleen.

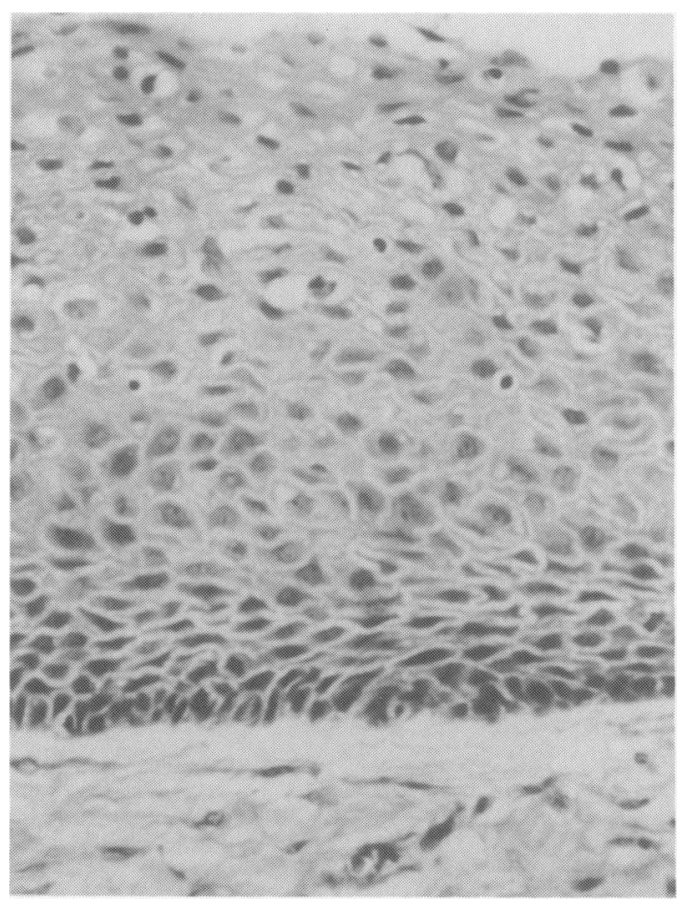

Figure 1 Microscopy of the splenic cyst showing florid epithelial cells (Case 1) (H\&E, × 26). 
An enlarged spleen weighing $297 \mathrm{~g}$ with a collapsed cyst measuring $19 \times 14 \times 9 \mathrm{~cm}$ with a corrugated and haemorrhagic internal surface was removed at laparotomy. Microscopically, the cyst was a primary epidermoid cyst with scanty fragments of atrophic squamous epithelium lining the inner surface of the sclerotic cyst wall.

\section{Discussion}

The most widely used classification of splenic cysts is that of Martin. ${ }^{4} \mathrm{He}$ classified them as primary (or true) cysts with cellular lining and secondary (or false) cysts without true cellular lining. He further classified primary cysts into parasitic and nonparasitic which may also be either congenital or neoplastic.

Epidermoid cysts are the commonest congenital cysts but still only account for $10 \%$ of the total of all splenic cysts. ${ }^{5}$ They are lined with stratified squamous epithelium but lack skin appendages which are seen in dermoid cysts. Macroscopically, the walls are greyish white and trabeculated. They contain serous fluid which is often blood stained.

Aetiologically they are thought to develop from mesonephrenic tissue included in the developing spleen in early embryonic life. The spleen lies close to the left mesonephrenic system and it has been suggested that abnormal development or trauma at the seventh week in utero may lead to inclusion of mesonephrenic elements within the spleen. ${ }^{6}$

The age at presentation ranges from newborns to 50 years, averaging around 17 years. They are slightly more common in women. ${ }^{7}$ Most epidermoid cysts are asymptomatic but can present as a painless or tender mass. Presentation as a surgical emergency is rare. ${ }^{8}$

Plain X-ray may show a soft tissue mass in the left upper quadrant displacing the stomach medially. A ring of calcification can often be seen. Abdominal ultrasound and computed tomographic scan are most valuable investigations. They usually show a circumscribed mass free from internal echoes. ${ }^{7}$

Traditionally, treatment was splenectomy as it was difficult to be certain of true diagnosis before operation. With increased accuracy of diagnostic tests and present attitudes towards splenic surgery, treatment is more conservative with preservation of the spleen if possible.

Popular techniques now developing along a more conservative line include drainage under radiographic control, de-roofing with external or internal peritoneal drainage, or simple cystectomy. ${ }^{6}$ These treatments should always be considered in children as the risk of pneumococcal infection is higher after splenectomy. However, conservative treatments should only be considered if infective causes have been excluded as peritoneal spread can occur with formation of multiple cysts.

\section{References}

1. Scott-Conner, C.E.H. The surgery of the spleen. In: Bardler, A.J. (ed.). The Spleen: Structures, Function and Clinical Significance. Chapman and Hall Medical, London, 1990, pp. 473-474.

2. Pappis, C.H., Demetriadis, D., Petrou, A. \& Pappis, H.C. Post-operative recurrence of splenic epithelial cyst; treatment with subtotal splenectomy. Z Kinder Chir 1990, 45: 245-248.

3. Ehrlich, R. \& Jamieson, C.G. Non-parasitic splenic cysts - a case report and review. Can J Surg 1990, 33: 306-308.

4. Martin, J.W. Congenital splenic cysts. Am J Surg 1958, 96: 302-308.

5. Todde, G., Bagolan, P., Fariello, G., Malena, S., Ferro, F. \& Modiello, G. Epidermoid cyst of the spleen in a newborn infant. Chir Pediatr 1989, 30: 172-174.

6. Carpenter, G., Cotter, P.W. \& Davidson, J.R. Epidermoid cyst of the spleen. Aust $N Z J$ Surg 1986, 56: 365-368.

7. Huang, T.Y., Wylie, R.R., Thomas, G. \& Cebedo, J. Epidermoid cyst of the spleen: a clinico-pathologic correlation. Indiana Med 1990, 83: 326-328.

8. Panossian, D.H., Wang, N., Reeves, C.D. \& Weeks, D.A. Epidermoid cyst of the spleen presenting as a generalised peritonitis. Am Surg 1990, 56: 295-298. 